\title{
The learning curve in head and neck reconstruction with microvascular free flaps: a retrospective review
}

\author{
Patnarin Mahattanasakul $^{\mathrm{a}}$, Veerachai Kerekhanjanarong ${ }^{\mathrm{a}}$, Porn-ake Apipan ${ }^{\mathrm{b}}$, Pakdee Sannikorn ${ }^{\mathrm{b}}$, \\ Pakpoom Supiyaphun ${ }^{\text {a }}$ \\ Affliations: \\ ${ }^{a}$ Department of Otolaryngology, Head and Neck Surgery, Faculty of Medicine, Chulalongkorn \\ University, Bangkok 10330, Thailand. ${ }^{b}$ The ENT Center of Excellence Rajavithi Hospital, Department \\ of Medical Services, Ministry of Health, Bangkok 10220, Thailand
}

\begin{abstract}
Background: Tissue defect around the head and neck region is a great challenge for reconstructive surgeons. Successful outcomes of surgery is influenced by many factors such as surgical technique in recipient and donor site, meticulous microvascular anastomosis, position of vascular pedicle, and post-surgery monitoring. The learning curve is important for any new microvascular surgeons.

Objectives: Evaluate the quality of training for a new otolaryngologist, head and neck surgeon, on their performance of free flaps in facial plastic and reconstructive surgery using a retrospective review.

Methods: Nineteen microvascular free flaps in patients from the ENT Center of Rajavithi Hospital were reviewed between November 2008 and October 2009. Incidences of free flap survival, and partial and complete necrosis were examined.

Results: Eighteen patients with microvascular free flaps had head and neck cancer. $88.9 \%$ of the patients had squamous cell carcinoma. There were nine radial forearm free flaps, five anterolateral thigh free flaps, two rectus abdominis free flap, two iliac-free flap, and one fibular-free flap. The overall success rate of the free flap reconstructions was $84 \%$. One partial and three total necrosis were done on the 19 flaps.

Conclusion: Causes of free flap failure were similar and not dependent on the experienced of the surgeons. The postgraduate training program in facial plastic and reconstructive surgery is effective in providing new microvascular surgeons the skills to perform successful microvascular and reconstructive surgeries.
\end{abstract}

Keywords: Free flaps, head and neck reconstruction, learning curve, microvascular surgery

In Asia, microvascular surgeries are not commonly performed in all hospitals except in medical universities or super tertiary referral centers. To have a successful outcome in microvascular surgery, several factors need to be considered. These are careful assessment of the patient before operation, atraumatic surgical technique in harvesting flap tissues and preparing the recipient's vessel for revascularization, perfect microvascular anastomosis, and positioning of pedicle to avoid tautness or kink in

Correspondence to: Veerachai Kerekhanjanarong, MD, Department of Otolaryngology, Head and Neck Surgery, Faculty of Medicine, Chulalongkorn University, Bangkok 10330, Thailand. E-mail: virachaik@yahoo.com the vascular pedicle [3].

Tissue defect around the head and neck region is a challenge for reconstructive surgeons. Even among experienced surgeons, the incidence of post-operative flap ischemia was $7-9 \%$ after free flap transfer and the overall rate of successful flap transfer was consistently around $94-95 \%[4,5]$.

Successful outcomes of surgery is influenced by many factors such as surgical technique in recipient and donor site, meticulous microvascular anastomosis, position of vascular pedicle, and post-surgery monitoring. The most important factor in avoiding free flap failure is the surgeon's operative experience [6]. Hence, the learning curve for all new microvascular surgeons is very important. 
The present study was designed to evaluate the quality of a new otolaryngologist-head and neck surgeon, in performing free flaps in facial plastic and reconstructive surgery using information from a retrospective review. The incidence of survival, partial and complete free flap necrosis were examined in detail.

\section{Materials and methods}

This retrospective case series reviewed the performance of the new otolaryngologist head and neck surgeon performing free flaps during the first year of fellowship in facial plastic and reconstructive surgery. Patients with head and neck defects that needed reconstructions were selected for this study. Eighteen patients who had microvascular surgery between November 2008 and October 2009 at the ENT Center of Excellence Rajavithi Hospital, Department of Medical Services, Ministry of Health, Thailand, were reviewed. Nineteen free flaps were done. This study was approved by the Ethics Committee of Royal College of Otolaryngology, Head and Neck Surgery, Thailand.

\section{Results}

Nineteen microvascular free flaps were performed in 18 patients. There were 14 males and four females with a mean age of 50.2 years (range: $38-71$ years). The patients' data is summarized in Table 1. Primary reconstruction was achieved in 17 microvascular free flaps $(89.7 \%)$. Secondary reconstruction was achieved in two microvascular free flaps $(10.5 \%)$. One of the cases that had secondary reconstruction was due to the failure of the pectoralis major flap from the primary reconstruction. The patient developed orocutaneous fistula and the wound became infected, resulting in necrosis. The area was debrided and secondary reconstruction was done by using the anterolateral thigh free flap. Contralateral recipient vessels of the neck were used to connect to the free flap. In the other case that had secondary reconstruction, the iliac crest free flap became infected. Secondary reconstruction was performed by using anterolateral thigh free flap.

All the patients in this study had head and neck cancer. $88.9 \%$ had squamous cell carcinoma. One case had adenoid cystic carcinoma while another had carcinoma ex pleomorphic. None of the cases received preoperative chemotherapy. However, one case had radiotherapy (70 Gy) before undergoing microvascular free flap surgery. Free flaps selected for reconstruction are listed in Table 1. The overall success rate of free flap reconstructions was $84.2 \%$ (16/19 flaps). Partial necrosis rate was 5.3\% (1/19 flaps) whereas the total necrosis rate was $15.8 \%$ (3/19 flaps). The results of microvascular free flap surgery are listed in Table 1.

Table 1. Patients demographic data, type of reconstruction and results of surgery.

\begin{tabular}{|c|c|c|c|c|c|c|c|}
\hline $\begin{array}{l}\text { Patient } \\
\text { No. }\end{array}$ & Age & Sex & Diagnosis & $\begin{array}{c}\text { Location } \\
\text { reconstruction }\end{array}$ & Type of free flap & $\begin{array}{c}\text { Pre-op } \\
\text { RT }\end{array}$ & $\begin{array}{l}\text { Result of } \\
\text { surgery }\end{array}$ \\
\hline 1 & 46 & $\mathrm{M}$ & CA buccal mucosa & Soft tissue & Radial forearm & no & Survive \\
\hline 2 & 48 & M & CA buccal mucosa & Soft tissue & Radial forearm & no & Partial necrosis \\
\hline 3 & 61 & $\mathrm{~F}$ & CA buccal mucosa & Soft tissue & Radial forearm & no & Survive \\
\hline 4 & 50 & $\mathrm{~F}$ & CA tongue & Soft tissue & Radial forearm & no & Survive \\
\hline 5 & 42 & $\mathrm{~F}$ & CA tongue & Soft tissue & Radial forearm & no & Survive \\
\hline 6 & 53 & M & CA tongue & Soft tissue & Rectus abdominis & no & Survive \\
\hline 7 & 47 & M & CA FOM & Osteocutaneous & Radial forearm & no & Survive \\
\hline 8 & 48 & M & CA supraglottic & Soft tissue & Radial forearm & no & Survive \\
\hline 9 & 48 & M & CA maxillary sinus & Soft tissue & Rectus abdominis & no & Total necrosis \\
\hline 10 & 71 & M & CA FOM & Osteocutaneous & Fibular & no & Survive \\
\hline 11 & 38 & M & Recurrent CA tongue & Soft tissue & Anterolateral thigh & 70 Gy & Survive \\
\hline 12 & 61 & M & CA tongue & $\begin{array}{l}\text { Osteocutaneous } \\
\text { Soft tissue }\end{array}$ & $\begin{array}{l}\text { Iliac } \\
\text { Anterolateral thigh }\end{array}$ & no & $\begin{array}{l}\text { Total necrosis } \\
\text { Survive }\end{array}$ \\
\hline 13 & 48 & M & CA tongue & Osteocutaneous & Iliac & no & Survive \\
\hline 14 & 58 & M & Adenoidcystic CA maxillary sinus & Soft tissue & Anterolateral thigh & no & Survive \\
\hline 15 & 46 & M & CA FOM & Soft tissue & Anterolateral thigh & no & Survive \\
\hline 16 & 49 & $\mathrm{~F}$ & CA tongue & Soft tissue & Radial forearm & no & Survive \\
\hline 17 & 42 & M & CA tongue & Soft tissue & Anterolateral thigh & no & Survive \\
\hline 18 & 48 & M & Carcinoma ex pleomorphic hard palate & Osteocutaneous & Radial forearm & no & Total necrosis \\
\hline
\end{tabular}

CA: carcinoma, FOM: Floor of mouth, RT = radiotherapy. 
All cases needed tracheostomy. Length of stay in the hospital ranged from 12 days to 57 days. Anesthesia time ranged from 315 minutes to 750 minutes (mean: 520.3 minutes), whereas the mean for flap ischemic time was 189.6 minutes.

Forty-nine anastomoses were performed. There were 19 arterial and 30 venous anastomoses. Most of the arterial anastomoses were performed in an end-to-end anastomotic fashion whereas four venous anastomoses were performed in an end-to-side fashion. There were 26 end-to-end venous anastomoses.

If a second vein was available, then it too would be anastomosed to provide an added measure of security. In this series, the superior thyroid artery and external jugular vein were the most frequently used as recipient vessels. Cervical anastomoses to the recipients' vessels are shown in Fig. 1.
There were three intraoperative complications. In one case, the radial artery was accidentally torn when the radial forearm free flap was being harvested. It was immediately repaired with a Nylon 9-0 and the flow of the radial artery was tested before anastomosis was performed. In another case, there was an intraoperative arterial thrombosis. The vessels were re-explored and thrombosis was removed before anastomosis was performed. In the last case, there was an intra-operative cephalic vein occlusion. The venous drainage had an abnormal blood flow. As a result of this, venae comitant was performed only in the venous anastomosis of the radial forearm free flap.

At the donor site, we had only one complication, we could not find the perforator branch in the anterolateral thigh free flap. Eventually, we abandoned the search for the perforator branch on this side of the leg and moved on to the other side of the leg. All

A

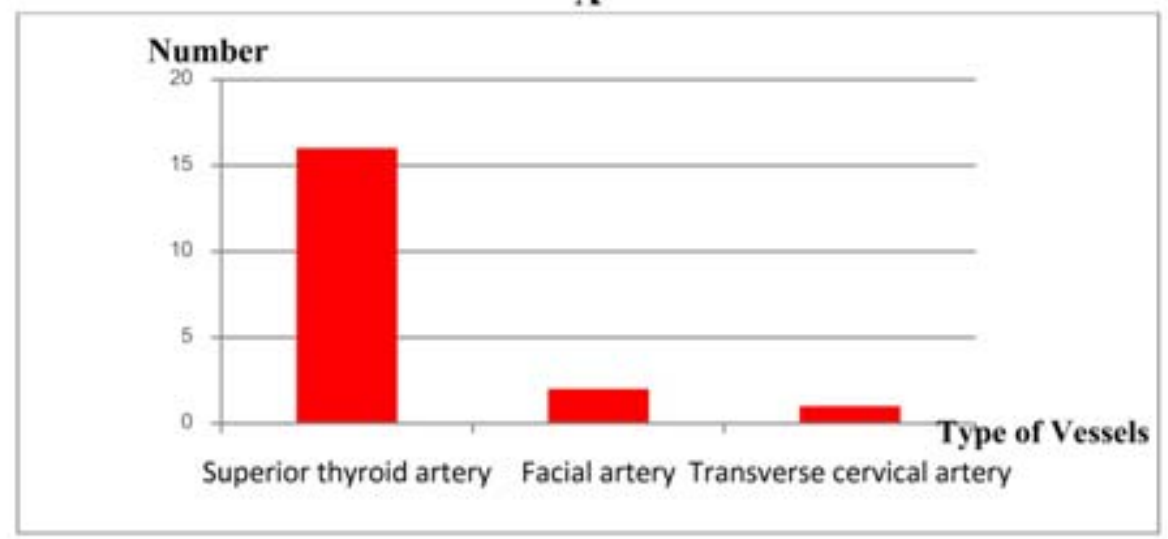

B

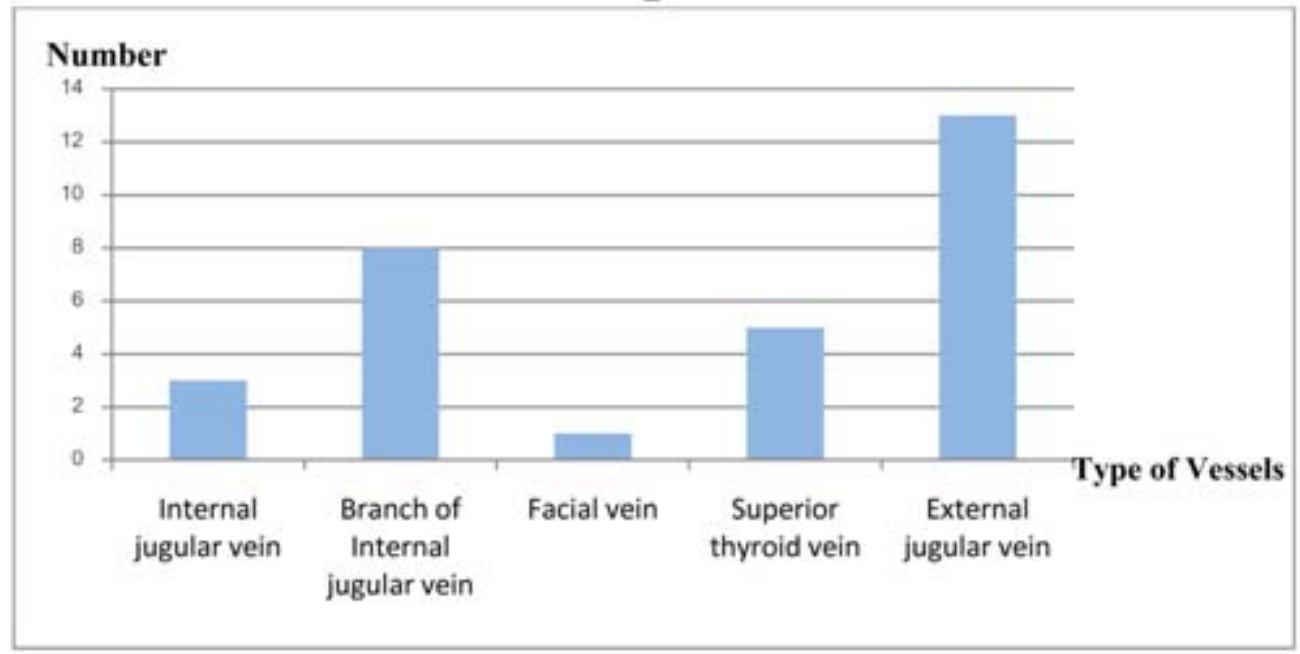

Fig. 1 Cervical anastomosis to the recipient artery (A) and vein (B). 
radial forearm free flaps and fibular free flaps at the donor sites were closed by split thickness skin graft. Primary closure technique was used to close donor sites for iliac free flaps, rectus abdominis free flap, and anterolateral thigh free flap. There were no major complications in any of the donor sites. There were only minor complications that required dressing and secondary wound healing.

As for the recipient site, two patients developed some complications when their residual tumor started to grow one month after their primary reconstruction (Table 2). In both cases, all efforts were made to resect all of the primary tumor. However, when total maxillectomy was performed, some tumors were left at the pterygomaxillary fossa. In the end, we had to send both patients for radiotherapy post-surgery. Minor complications of the recipient sites were managed by dressing the wounds, debridement, incision, and drainage at the minor operating theatre. Wound dehiscent and orocutaneous fistula were resutured with local anesthesia.

Table 2. Post-operative complications.

\begin{tabular}{lc}
\hline Complications at the recipient site & Number \\
\hline Flap total necrosis & 3 \\
Flap partial necrosis & 1 \\
Infected wound & 6 \\
Wound dehiscent & 1 \\
Seroma & 3 \\
Orocutaneous fistula & 3 \\
Hematoma & 1 \\
Residual tumor & 2
\end{tabular}

In this study, there was no surgical mortality. Seven patients had systemic complications; postoperative pneumonia was the most common.

\section{Discussion}

In this retrospective study, 18 patients had reconstructive surgery using free-flaps after having radical ablative cancer surgery in the head and neck where the success rate was approximately $85 \%$. We examined 19 series conducted during one year of microvascular training, where the success rate was comparable to that obtained at three different institutes in facial plastic and reconstructive surgery by Blackwell et al. [3]. Our overall success rate during the first year of practice was comparable to those reported by experienced surgeons.
The number of studied cases is small, but the success rate will increase with more experience. For example, Urken et al. [5] reported an $89.0 \%$ success rate after performing 75 free flaps and after another 125 cases, the success rate increased to $96.0 \%$. Watkinson and Breach [7] reported a 79.0\% success rate after performing 64 microvascular free flaps and after another 77 more cases, the success rate was up to $95.0 \%$. Godina et al. [8] reported a $74.0 \%$ success rate after 100 free flaps and after another 100 free flaps, the success rate was $96.0 \%$. Harashima et al. [9] also reported a $75.0 \%$ success rate after performing free-flaps in his first three years as compared to an improved success rate of $97.0 \%$ the next five years. Aside from that, the success rate has been shown to be positively correlated with number of cases performed over time. For instance, two independent studies reported a $94.0 \%$ and $92.3 \%$ success rate after performing 479 and 540 free-flaps over the span of 18 years, respectively $[10,11]$.

In our study, the success rate of primary reconstruction was $89.7 \%$. This was comparable to a success rate of $96.3 \%$ reported by Gonzalez-Garcia et al. [12]. One of the benefits of doing primary reconstruction is the absence of fibrosis in the surgical bed, easy access to the recipient's vessels, and no affect on the patient's functional ability. Furthermore, the most commonly used flap is the radial forearm flap. The reason for this is that the vessels are usually of high caliber (artery: 2-3 mm, cephalic vein: 3-4 mm, deep veins: $1-3 \mathrm{~mm}$ ), and the vascular pedicles are typically longer [13]. The risk for free-flap failure increases when the pedicle diameter is less than $1 \mathrm{~mm}$ [6]. Successful microvascular surgery is dependent on the use of a long vascular pedicle. It has been shown using a multivariate analysis [14] that the use of vein graft to lengthen the vascular pedicle is correlated with an increased risk of having freeflap failure. However, this was not our case because in one patient, the rectus abdominis free-flap failed due to tension of anastomosis between inferior epigastric vein and the internal jugular vein. For this patient, a secondary reconstruction had to be done by using an anterolateral thigh free-flap. In fact, external jugular vein graft was used to lengthen the venous vessel to avoid creating tension of the vascular pedicle.

Along the same lines, imperfect microvascular techniques such as kinking of the vascular pedicle are the most common cause for free-flap failure among inexperienced surgeon [10]. In our series, one of the 
radial forearm free-flap failed because of a kink and external compression from palatal obtulator. The radial forearm free-flap was debrided and replaced. Another case of our failure was due to infection. Iliac freeflap was infected with Pseudomonas aeruginosa and thrombosis developed. Then, debridement was done, and forehead flap was used instead. Unfortunately, the forehead flap became necrotic because the donor's vascular pedicle became malfunctional. Therefore, for the reconstruction, anterolateral thigh free-flap was done post-surgery on day 34 . The recipient vessels from the contralateral neck were used instead.

Another post-operative complication in our study was wound infection. Head and neck reconstruction are commonly connected between the oral cavity, nose and paranasal sinus. Hence, they are easily contaminated by saliva and secretion. Orocutaneous fistula was reported as the most common woundinfection, where out of 38 radial forearm free-flaps, three cases had fistulas [15]. In our study, all of the fistulas were debrided and resutured in the minor operating theatre.

In our retrospective review, cases that had unsuccessful flaps are very useful for new surgeons as a learning tool to avoid mistakes in microvascular surgery. One limitation of the present study is the method of measurement used to assess the success of reconstruction. Partial or total free-flap necrosis is relatively basic if we consider other factors such as the result of treatment, donor site morbidities, and impaired cosmetic and functional outcome. Another limitation of our study is our small number of cases. For this reason, we were unable to have a multiinstitutional analysis. Nevertheless, our study confirms that a learning curve in microvascular head and neck surgery can be achieved through fellowship training and that learning is an ongoing process.

In conclusion, postgraduate training program in facial plastic and reconstructive surgery appears to be effective in giving new microvascular surgeons opportunities to perform microvascular and reconstructive surgery. This training has helped inexperienced surgeons avoid some of the most common mistakes that affect the success rate of flaps. We were able to achieve a high success rate of freeflap transfer.

\section{Acknowledgments}

We would like to thank all of the staffs and residents at ENT Center of Excellence Rajavithi
Hospital. The authors have no conflict of interest to declare.

\section{References}

1. Stiefel M, Shaner A, Schaefer SD. The Edwin Smith Papyrus: the birth of analytical thinking in medicine and otolaryngology. Laryngoscope. 2006; 116:182-8.

2. Whitaker IS, Karoo RO, Spyrou G, Fenton OM. The birth of plastic surgery: the story of nasal reconstruction from the Edwin Smith Papyrus to the twenty-first century. Plast Reconstr Surg. 2007; 120: 327-36.

3. Blackwell KE, Brown MT, Gonzalez D. Overcoming the learning curve in microvascular head and neck reconstruction. Arch Otolaryngol Head Neck Surg. 1997; 123:1332-5.

4. Hildago DA, Jones CS. The role of emergent exploration in free tissue transfer: a review of 150 consecutive cases. Plast Reconstr Surg. 1990; 86: 492-98.

5. Urken ML, Weinberg H, Buchbinder D, Moscoso JF, Lawson W, Catalano PJ, et al. Microvascular free flaps in head and neck reconstruction: Report of 200 Cases and Review of Complications. Arch Otolaryngol Head Neck Surg. 1994; 120:633-40.

6. Khouri RK, Avoiding free flap failure. Clin Plast Surg. 1992;19:773-81

7. Watkinson JC, Breach NM. Free flaps in head and neck reconstructive surgery: a review of 77 cases. Clin Otolaryngol. 1991; 16:350-53.

8. Godina M, Early Early microsurgical reconstruction of complex trauma of the extremities. Plast Reconstr Surg. 1986; 78:285-99.

9. Harashina T. Analysis of 200 free flaps. Br J Plast Surg. 1988;41:33-6.

10. Eckardt A, Fokas K. Microsurgical reconstruction in the head and neck region: an 18-year experience with 500 consecutive cases. J Craniomaxillofac Surg. 2003; 31:197-201.

11. Pohlenz P, Blessmann M, Blake F, Li L, Schmelzle R, Heiland M. Outcome and complications of 540 microvascular free flaps: the Hamburg experience. Clin Oral Investig. 2007; 11:89-92.

12. Gonzalez-Garcia R, Rodriguez-Campo FJ, Naval-Gias L, Sastre-Perez J, Munoz-Guerra MF, Usandizaga JL, et al. Radial forearm free flap for reconstruction of the oral cavity: clinical experience in 55 cases. Oral Surg Oral Med Oral Pathol Oral Radiol Endod. 2007; 104:29-37.

13. Wolf KD, Holzle F, editors. Raising of microvascular 
flaps a systematic approach. $1^{\text {st }}$ ed. Berlin: Springer; 2005.

14. Tsuji H, Minami T, Yamashita T, Kumazawa T. Head and neck reconstruction with free flap transfer and its surgical indications: our experience. Acta Otolaryngol. 1993; 500:131-34.
15. Chen CM, Lin GT, Fu YC, Shieh TY, Huang IY, Shen YS, Chen CH. Complications of free radial forearm flap transfer for head and neck reconstruction. Oral Surg Oral Med Oral Pathol Oral Radiol Endod. 2005; 99:671-6. 